\title{
The assessment of space radiation exposure of biology experiments during two short-term missions
}

\author{
Mariagabriella Pugliese ${ }^{1,2, *}$, Filomena Loffredo ${ }^{1,2}$, Maria Quarto ${ }^{1,2}$, Vincenzo Roca ${ }^{1,2}$, \\ Gianna Vivaldo ${ }^{3}$, Alba Zanini ${ }^{3}$ \\ ${ }^{1}$ Dipartimento di Fisica, Università degli Studi di Napoli Federico II, Naples, Italy \\ ${ }^{2}$ Istituto Nazionale di Fisica Nucleare (INFN), Sezione di Napoli, Naples, Italy \\ ${ }^{3}$ Istituto Nazionale di Fisica Nucleare (INFN), Sezione di Torino, Turin, Italy
}

\section{Email address:}

pugliese@na.infn.it (M. Pugliese)

\section{To cite this article:}

Mariagabriella Pugliese, Filomena Loffredo, Maria Quarto, Vincenzo Roca, Gianna Vivaldo, Alba Zanini. The Assessment of Space Radiation Exposure of Biology Experiments during Two Short-Term Missions. American Journal of Environmental Protection.

Vol. 3, No. 6, 2014, pp. 323-326. doi: 10.11648/j.ajep.20140306.14

\begin{abstract}
The assessment of space radiation exposure on plants, animals, cell cultures, as well as the astronauts, is crucial not only because it is very different from that to which they are subjected on the earth, but also to understand the combined effects of exposure to space radiation under microgravity. In particular, we evaluated the equivalent dose due to neutrons and charged particles for biology experiments, in two short-term missions, FOTON M3 and STS-34 mission, using Thermo Luminescence Dosimeters (TLDs) to evaluate the charged particles dosimetry and a set of passive neutron bubbles detectors for neutron dosimetry. In STS-34 mission, stack bismuth track dosimeter was used in addition to bubbles detectors to evaluate the dose equivalent rate due to neutrons in a wide energy range $(0.025 \mathrm{eV}-200 \mathrm{GeV})$. Considering a total dose due to both radiation types particles, a value of about $1 \mathrm{mSv} /$ day is obtained. The neutron component represents about $18 \%$ of the total.
\end{abstract}

Keywords: Space Radiation, Dosimetry

\section{Introduction}

Microgravity is associated with the disturbance of a number of parameters at both organ and cellular levels and many studies have been conducted to evaluate if the radiosensitivity is modified under microgravity conditions [1]. In fact microgravity and radiation are the two main factors acting on biological systems during space flights. Since the beginning of the space era the idea of investigating biological phenomena in microgravity has triggered the interest of scientists and the scientific community.

So it is very important to assess the exposure of all being to the mixed field of space radiation. Obviously, the experiments in space represent the ideal benchmark to test the effects of weightlessness on the processing of radiation-induced damage compared to ground-based radiobiology experiments, and for this reason flight opportunities are very important. In addition, the measurements made for biology experiments, will also be useful to assess the exposure of astronauts to space radiation.

The radiation environment inside a spacecraft is very complex, because it is due to the interaction of primary radiation (electrons, protons and heavy ions from GCR and Van Allen Belts) with the shielding materials that produces a shower of secondary particles. In particular, the assessment of the neutron dose requires different detectors, because of their energy spectrum, ranged from thermal energies to several hundred of GeV.

The unmanned FOTON M3 and the STS-134 missions represented two short-term flight opportunities to evaluate the effect of microgravity in different experiments, accommodated inside BIOKON, a KI (Kayser Italia) container for experiments processing (see Fig. 1). BIOKON provides a dedicated environment for the execution of life science experiments in microgravity. BIOKON complements effectively all types of biological Experiment Units (EU) regardless the fact that they could require electrical power or not [2].

The radiation detector systems applied for the monitoring of the radiation environment onboard the International Space Station (ISS) include passive and active detectors [3]. Passive detectors are largely used to determine the space radiation exposure of the experiments because they are easy to handle, 
comparatively light-weight, do not require power consumption, and give integral information about dose by post-flight evaluation [4]. These features are clearly essential in case of unmanned mission, as FOTON M3 mission. The dose contribution due to charged particles was evaluated using three different types of TLDs: TLD 100 (LiF:Mg,Ti), TLD 600 ( $\left.{ }^{6} \mathrm{LiF}: \mathrm{Mg}, \mathrm{Ti}\right)$ and TLD $700\left({ }^{7} \mathrm{LiF}: \mathrm{Mg}, \mathrm{Ti}\right)$, while neutron dosimetry was performed by bubble dosimeters (BTI Ontario, Canada) from thermal energy to $20 \mathrm{MeV}$, Polycarbonate detectors from $20 \mathrm{MeV}$ to $100 \mathrm{GeV}$ and Bismuth stack tracks detectors over $20 \mathrm{MeV}$ to $200 \mathrm{GeV}$ [5].

Two dosimeters for each type were hosted inside a plastic box to record the radiation exposure during all the prelaunch and return phases. The readings of these detectors were subtracted from the readings of the on-flight detectors to evaluate only the radiation in orbit.

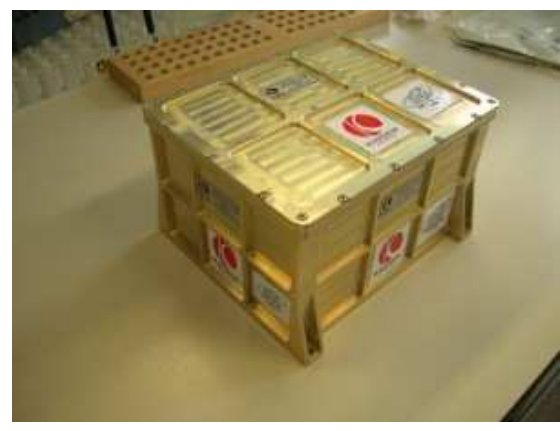

Figure 1. BIOKON container

\section{Flight Opportunities}

\subsection{FOTON M3 Mission}

FOTON -M is a series of robotic spacecraft used by Russia and the European Space Agency (ESA) for research conducted in the microgravity environment of Earth orbit (see Fig. 2). The unmanned FOTON-M3 spacecraft was launched on September 142006 from Baikonur Cosmodrome, in Kazakhstan and spent 12 days in low-Earth orbit. The FOTON capsule carries a 400 $\mathrm{kg}$ European experiment payload with experiments in a range of scientific disciplines - including fluid physics, biology, crystal growth, radiation exposure and exobiology.

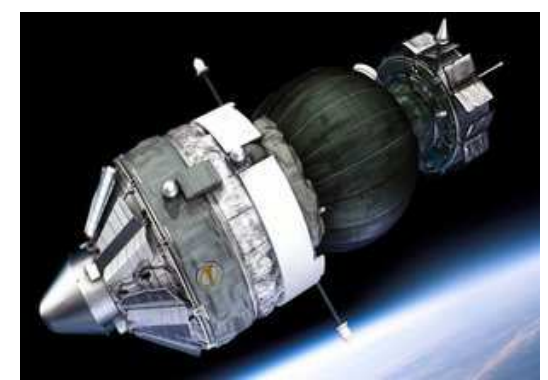

Figure 2. FOTON-M Capsule

During the flight the European experiments and equipment are monitored by ESA's Operations Team at the Payload Operations Centre based at Esrange near Kiruna, Sweden. They are responsible for receiving, evaluating and disseminating scientific data generated by European payloads on FOTON. The team also receive support from two ground stations in Canada.

\subsection{STS-134 Mission}

STS-134 mission was the latest of the shuttle Endeavour (see Fig. 3). The Italian Space Agency led on board the STS-134 Space Flight of Roberto Vittori a program of experiments, that involved the interest and resources of a number of different actors from institutions, international space agencies, scientific communities, industry and society to promote the exploitation of the International Space Station. The mission lasted for 16 days, from May 16 to the 1 st June 2011.

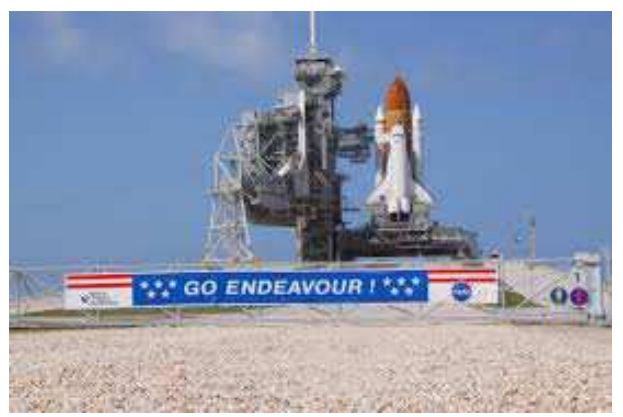

Figure 3. STS-134 Space Shuttle launch

In these short-term missions, has been exploited the microgravity condition and they represented a good flight opportunity to carry out research activities ranging from material science to electronics, from cellular biology to radiation protection, from chemistry to medical prognosis.

During the last STS-134 Endeavour mission, the BIOKON took part into the DAMA mission promoted by the Italian Space Agency (ASI). On the whole, seven different Italian experiments designed both in the domain of biological sciences and dosimetry were integrated in two BIOKONs.

\section{Materials and Methods}

\subsection{Charged Particle Dosimetry}

A thermo Luminescent Dosimeter measures ionizing radiation exposure by measuring the intensity of visible light emitted from a crystal in the detector when heated. The intensity of light emitted is dependent upon the radiation exposure.

Although GCR consist of $85 \%$ protons, $14 \%$ helium, and $1 \%$ heavier particles (iron $0,02 \%$ only), HZE nuclei are very important in space radiation dosimetry because highly charged and very densely ionizing. As a consequence, even though the number of HZE particles is relatively small, they have a significant biological impact that is comparable to that of protons.

Measurements to evaluate the charged particles dosimetry in these two short-term flights were obtained using three different types of TLD: TLD 100 (LiF:Mg,Ti), TLD 600 
(6LiF:Mg,Ti) and TLD 700 (7LiF:Mg,Ti), previously calibrated at the NASA Space Radiation Laboratory (NSRL) at the Brookhaven National Laboratory, Upton New York [6]. The main purpose of these ground based measurements was to acquire TL efficiency to apply this knowledge to the determination of equivalent dose from space radiation. Since the energy range of space radiation ranges up to a few $\mathrm{GeV} / \mathrm{n}$ and its spectral composition extends from protons to Fe nuclei, evaluation of TL-efficiency is crucial to the performance of TLDs [7]. The relative TL-efficiency, $\eta$ (E), describes quantitatively the efficiency of conversion of radiation energy absorbed in the detector into TL light, as compared with efficiency of such conversion after with standard radiation $(\gamma$ radiation):

$$
\eta(E)=\frac{T L(E) / D(E)}{T L(\gamma) / D(\gamma)}
$$

Our interest was to determine TL efficiencies, because it depends not only upon the LET but also on the ion charged.

TLDs were annealed in air at $400^{\circ} \mathrm{C}$ for $1 \mathrm{~h}$ before the exposition. Post-flight reading of the TLDs was performed by an Harshaw model 3500 manual TLD reader.

\subsection{Neutron Dosimetry}

Neutrons are a not avoidable component of the secondary radiation produced by interaction of primary radiation with the spacecraft shielding. Because of their high LET, neutrons could represent a main risk for both the electronic instruments and the health of the astronauts during space missions. For this reason, in both missions, the neutron component has been separately evaluated.

A set of passive detectors has been used: two types of bubble detectors (BTI Ontario, Canada), BDT for thermal neutrons (0.025 eV- $4 \mathrm{eV}), \mathrm{BD}-\mathrm{PND}$ for fast neutrons (100 keV-20 MeV), polycarbonate detectors $(20 \mathrm{MeV}-100 \mathrm{MeV})$ and ${ }^{209} \mathrm{Bi}$ stack $(20 \mathrm{MeV}-200 \mathrm{GeV})$. Because of space and weight limits, only bubble detectors BD-PND, sensitive in the energy interval 100 $\mathrm{keV}-20 \mathrm{MeV}$, are used in FOTON M3 mission.

In the STS-134 mission the entire detector set has been used and with the elaboration of the detector readings using the unfolding code BUNTO [8], the neutron energy spectrum has been obtained. The equivalent dose rate has been evaluated in the energy interval $0.025 \mathrm{eV}-200 \mathrm{GeV}$.

\section{Results}

\subsection{FOTON M3 Mission}

The re-entry module, a $2.2 \mathrm{~m}$ diameter sphere, was the only retrievable part of the satellite. The FOTON-M3 mission took place during the minimum phase of the 23rd solar cycle; therefore, the satellite flew during a period characterized by very low solar activity, without the occurrence of any high-energetic solar phenomena such as flares, Coronal Mass Ejections and Solar Energetic Particle events. Thus, the space radiation environment at the Earth orbit can be considered as stable during the mission.

During this mission, TLDs were allocated into three different BIOKON (1, 4 and 5). The BIOKON 1 was dedicated to dosimetry experiments for the study of the effectiveness of different shields in the reduction of the dose, while the BIOKON 4 and 5 hosted the TLDs to assess the dose for biology experiments. The equivalent dose rate due to charged particles was obtained using TLD 700. The results are shown in tab.1 [9].

Neutron dosimetry was performed using BD-PND bubble detectors, sensitive in the energy range (100 keV -20 MeV). Three detectors were placed on the top of Photo-II optical sensor system, a high-tech system which measured in real time the chlorophyll fluorescence to study the effects of the space ionizing radiation and microgravity on several photosynthetic microorganisms [10]. For this mission, a simulation of the entire process of primary radiation interaction and production of secondary neutrons inside the satellite has been carried out and the various components of the secondary radiation have been separately calculated with GEANT4 MC code.

FOTON M3 capsule geometry was modeled as a sphere of external radius $1130 \mathrm{~mm}$ and composed of three concentric shells in Carbon fibre $(5 \mathrm{~cm})$, Aluminum $(3.3 \mathrm{~cm})$ and Kevlar $(3.7 \mathrm{~cm})$ [11]. The experimental value obtained with BD-PND detectors is in good agreement with the calculated one (see Table 1).

Table 1. Equivalent dose rate during FOTON M3 mission

\begin{tabular}{lllll}
\hline Conteiner & Detector & Particle & Energy & Equivalent dose rate $(\boldsymbol{\mu S v} /$ day $)$ \\
\hline BIOKON 1, 4,5 & TLD 700 & Charged Particles & All energies & $520 \pm 43$ \\
Photo II & BD-PND & Neutrons & $10 \mathrm{keV}-20 \mathrm{MeV}$ & $73 \pm 2083 \quad 8$ with GEANT4 \\
\hline
\end{tabular}

\subsection{STS-134 Mission}

In Table 2 the results for all types of TLDs exposed on board of the Shuttle are reported. Errors are standard deviations. The value of the dose equivalent rate ( $\mu$ Sv/day) is the same for TLD 100 and TLD 700, and highest for TLD 600 that are sensitive also to neutrons.

Table 2. Charged particles equivalent dose rate

\begin{tabular}{ll}
\hline TLD type & Equivalent dose rate $(\boldsymbol{\mu S v} /$ day $)$ \\
\hline TLD 100 & 775 \\
TLD 600 & 870 \\
TLD 700 & 772 \\
\hline
\end{tabular}


The neutron ambient equivalent dose has been evaluated in a wide energy range $(0.025 \mathrm{eV}-200 \mathrm{GeV})$ by using the entire detector set above described. In table 3 the results are reported.

Table 3. Neutron ambient equivalent dose rate

\begin{tabular}{ll}
\hline TLD type & Equivalent dose rate $(\boldsymbol{\mu S v} /$ day $)$ \\
\hline $0.025 \mathrm{eV}-10 \mathrm{keV}$ & 14 \\
$10 \mathrm{keV}-20 \mathrm{MeV}$ & 94 \\
$20 \mathrm{MeV}-200 \mathrm{GeV}$ & 67 \\
Total & 175 \\
\hline
\end{tabular}

\section{Conclusions}

From the comparison of the results obtained from the neutron dosimetry and those obtained with TLD 600 (subtracting the contribution of these TLD 700), it was observed that the values obtained for neutron dosimetry are higher, this is due to the fact that the TLD 600 are only sensitive to thermal neutrons [12]. The total equivalent dose rate is about $1 \mathrm{mSv} /$ day, in agreement with the data reported in the literature. In fact models of the space environment outside of LEO have predicted that astronaut crews can receive a total body dose of approximately 1-2 $\mathrm{mSv}$ per day in interplanetary space and approximately $0.5-1 \mathrm{mSv}$ each day on the surface of Mars due to GCR radiation.

\section{References}

[1] R. D. Morrison, "Cellular changes in microgravity and the design of space radiation experiments", Adv. Space Res. 14, 1994, pp. 1005-1019.

[2] M. Vukich, P. L. Ganga, D. Cavalieri, L. Rizzetto, D. Rivero, S. Pollastri, S. Mugnai, S. Mancuso, S. Pastorelli, M. Lambreva, A. Antonacci, A. Margonelli, I. Bertalan, U. Johanningmeier, M.T. Giardi, G. Rea, M. Pugliese, M. Quarto, V. Roca, A. Zanini, O. Borla, L. Rebecchi, T. Altiero, R. Guidetti, M. Cesari, T. Marchioro, R. Bertolani, E. Pace, A. De Sio, M. Casarosa, L. Tozzetti, S. Branciamore, E. Gallori, M. Scarigella, M. Bruzzi, M. Bucciolini, C. Talamonti, A. Donati, V. Zolesi, "BIOKIS: a model payload for multidisciplinary experiments in microgravity”, Microgravity Sci. Tec. 24, 2012, pp.397-409.

[3] L. Narici, F. Belli, V. Bidoli, M. Casolino, M. P. De Pascale, L. Di Fino, G. Furano, I. Modena, A. Morselli, P. Picozza, E. Reali, A. Rinaldi, D. Ruggirei, R. Sparsoli, V. Zaconte, W.G. Sannita, S. Carozzo, S. Licoccia, P. Romagnoli, E. Traversa, V. Cotronei, M. Vazquez, J. Miller, V.P Salnitskii, O.I. Shevchenko, V.P. Petrov, K.A. Trukhanov, A. Galper, A. Khodarovich, M.G. Korotkov, A. Popov, N. Vavilov, S. Avdeev, M. Boezio, W.
Bonvicini, A. Vacchi, N. Zampa, G. Mazzenga, M. Ricci, P. Spillantini, G. Castellini, R. Vittori, P. Carlson, C. Fuglesang, D. Schardt, "The ALTEA/ALTEINO projects: studying functional effects of microgravity and cosmic radiation", Adv. Space Res. 33 vol.8, 2004, pp.1352-1357.

[4] P. Bilski, "Dosimetry of densely ionising radiation with three LiF phosphors for space applications", Rad. Prot. Dos. 120, 2006, pp. 397-400.

[5] W.G. Cross, L. Tommasino, "Dosimetry of high energy neutrons and protons by Bi-209 fissions", Rad. Prot. Dos. 70, 1997, pp. 419-424.

[6] M. Pugliese, V. Roca, M. Durante, "The use of TL dosimeters in HZE radiation fields", in Proceedings of Third European IRPA Congress, 14-18 June, Helsinki, Finland., 2010, pp. 4-18.

[7] T. Berger, M. Hajek, L. Summerer, M. Fugger, N. Vana, "The effciency of various thermoluminescence dosemeter types to heavy ions", Rad. Prot. Dos. 120, 2006, pp. 365-368.

[8] A. Zanini, M. Storini, O. Saavedra, "Cosmic rays at High Mountain Observatories”, Adv. Space Res., 44 vol.10, 2009, pp. 1160-1165.

[9] M. Pugliese, V. Bengin, M. Casolino, V. Roca, A. Zanini, M. Durante, "Tests of shielding effectiveness of Kevlar and Nextel onboard the International Space Station and the Foton-M3 capsule", Rad. Environ. Biophys. 49, 2010, pp. 359-363.

[10] G. Rea, D. Esposito, M. Damasso, A. Serafini, A. Margonelli, C Faraloni, G. Torzillo, A. Zanini, I. Bertalan, V. Johanningmeier, M.T. Giardi, "Ionizing radiation impacts photochemical quantum yield and oxygen evolution activity of photosystem II in photosynthetic microorganisms", Inter. J. Rad. Biol. 84 vol.11, 2008, pp. 867-877.

[11] M. Damasso, T. Dachev, M.T. Giardi, G. Falzetta, G. Rea, A. Zanini, "Experimental data and GEANT4 Monte Carlo predictions of the radiation environment on board Foton-M3 satellite," in Proceedings Workshop Fundamental Space Research, 23-28 September, Sunny Beach, Bulgaria, 2008, pp.159-162.

[12] M. Pugliese, F. Loffredo, M. Quarto, V. Roca, C. Mattone, O. Borla, A. Zanini, "Results of nDOSE and HiDOSE Experiments for Dosimetric Evaluation During STS-134 Mission”, Microgravity Sci. Tec. 25, 2014, pp. 353-358.

[13] G. D.Badhwar, W. Atwell, F. F. Badavi, T. C. Yang and T. F. Cleghorn, "Space Radiation Absorbed Dose Distribution in a Human Phantom", Radiat. Res. 157, 2002, pp.76-91. 